\title{
Collapse of Orthotropic Spherical Shells
}

\section{Journal Article}

\section{Author(s):}

Munglani, Gautam; Wittel, Falk K. (1); Vetter, Roman (Di); Bianchi, Filippo; Herrmann, Hans J.

Publication date:

2019-08-02

Permanent link:

https://doi.org/10.3929/ethz-b-000358596

\section{Rights / license:}

In Copyright - Non-Commercial Use Permitted

\section{Originally published in:}

Physical Review Letters 123(5), https://doi.org/10.1103/PhysRevLett.123.058002

\section{Funding acknowledgement:}

319968 - Fluid Flow in Complex and Curved Spaces (EC) 


\title{
Collapse of Orthotropic Spherical Shells
}

\author{
Gautam Munglani, ${ }^{1,2,{ }^{*}}$ Falk K. Wittel, ${ }^{1}$ Roman Vetter, ${ }^{1}$ Filippo Bianchi, ${ }^{1}$ and Hans J. Herrmann ${ }^{1,3}$ \\ ${ }^{1}$ Computational Physics for Engineering Materials, Institute for Building Materials, ETH Zürich, \\ Stefano-Franscini-Platz 3, CH-8093 Zürich, Switzerland \\ ${ }^{2}$ Institute of Plant and Microbial Biology, University of Zürich, Zollikerstrasse 107, CH-8008 Zürich, Switzerland \\ ${ }^{3}$ Physique et Mécanique des Milieux Hétérogènes (PMMH), ESPCI, 7 quai St. Bernard, 75005 Paris, France
}

(Received 28 February 2019; revised manuscript received 24 June 2019; published 31 July 2019)

\begin{abstract}
We report on the buckling and subsequent collapse of orthotropic elastic spherical shells under volume and pressure control. Going far beyond what is known for isotropic shells, a rich morphological phase space with three distinct regimes emerges upon variation of shell slenderness and degree of orthotropy. Our extensive numerical simulations are in agreement with experiments using fabricated polymer shells. The shell buckling pathways and corresponding strain energy evolution are shown to depend strongly on material orthotropy. We find surprisingly robust orthotropic structures with strong similarities to stomatocytes and tricolpate pollen grains, suggesting that the shape of several of Nature's collapsed shells could be understood from the viewpoint of material orthotropy.
\end{abstract}

DOI: 10.1103/PhysRevLett.123.058002

The collapse of spherical shells is as ubiquitous in Nature and technology as it is strikingly diverse, producing a myriad of postbuckled shapes when the delicate balance between geometry, material properties, and kinetics is perturbed. Understanding this behavior is necessary for a multitude of applications ranging from the prevention of catastrophic failure in water tanks and submarines [1,2], and the production of functional colloids [3-5], to ensuring the survival of pollen during dispersal by harmomegathy [6,7], and predicting healthy human blood cell shapes [8,9]. Factors like the Poisson ratio [10,11], transverse shear and effective bending stiffness [12], viscoelasticity [5], and compression rate [13] have already been implicated in controlling the buckling pathways and postbuckling conformations for homogeneous spherical shells. Furthermore, inhomogeneities like variable cell wall thickness [14], local soft spots [15], and spheroidal geometries [16] exert an even stronger influence in directing collapse. Computer simulations, along with nonlinear shell theory [17-19] have proved invaluable in elucidating the collapse mechanics of isotropic spherical shells $[4,10,13]$. However, shells with anisotropic properties, a common feature of composite materials like biological cell walls [20,21], have also been simulated by applying identical isotropic assumptions, with mixed results [11]. So far, material anisotropy as a potential key player in shell morphogenesis has been largely excluded from thin shell analyses.

In this Letter, a combination of numerical and experimental approaches are used to reveal the diversity of tunable and robust postbuckled conformations produced by the controlled collapse of in-plane orthotropic spherical shells. We find that adjusting the ratio of elastic properties between orthogonal directions and varying the slenderness of the shell alters the strain energy landscape of the collapse. This material irregularity therefore regulates the initial buckling and subsequent folding pathway, driving the system towards three distinct classes of conformations based largely on the degree of orthotropy. We find that there is a material regime in which orthotropic spherical shells can be more stable than isotropic ones under volumecontrolled collapse. Finally, we present the scaling of the critical pressure at which buckling is initiated depending on these two control parameters. With this new insight into the stability of spherical shells, research on the morphogenesis of pollen grains, red blood cells, gel phase vesicles, blastulae, and related structures is offered a new perspective in explaining the rich variety of observed shapes.

Thin isotropic spherical shells of radius $R$, wall thickness $h$, Young's modulus $E$, and Poisson ratio $\nu$, have been shown to collapse under a critical external pressure $p_{\mathrm{ci}}$ in a process resembling a first-order transition given by [22,23]

$$
p_{\mathrm{ci}}=\frac{4 E}{\sqrt{12\left(1-\nu^{2}\right)}}\left(\frac{h}{R}\right)^{2} .
$$

$p_{\text {ci }}$ is inversely proportional to the square of the shell slenderness, which we define as $R / h$. When subjected to an increasing uniform external pressure $p<p_{\text {ci }}$, the spherical shell contracts isotropically, resulting in increased elastic energy due to in-plane compression, while maintaining a constant bending energy. When $p_{\mathrm{ci}}$ is reached, the compressive stress is released at the cost of a transverse deflection in the form of a local indentation [Fig. 1(a)]. In anisotropic shells, some areas are more prone to grow inwards than others, leading to symmetry breaking and a lower critical pressure, $p_{\mathrm{c}}$. 

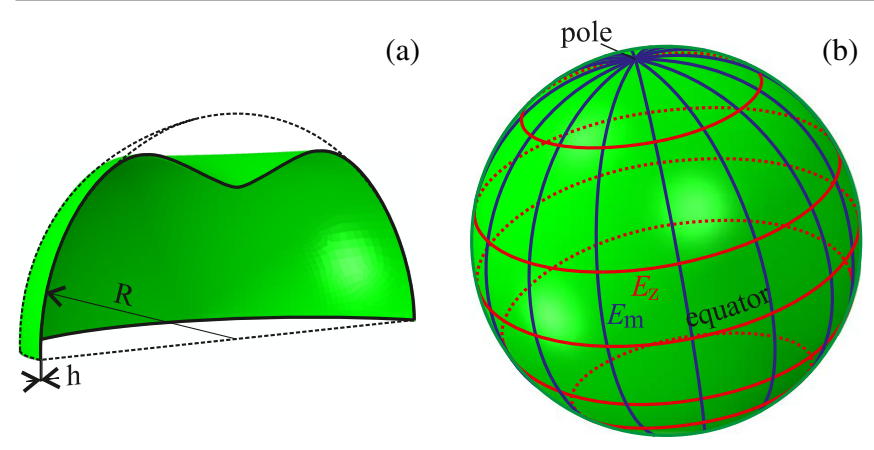

FIG. 1. (a) Schematic representation of a single indentation on a spherical shell with radius $R$ and thickness $h$. (b) Illustration of the meridional and zonal directions on the spherical shell with their moduli $E_{\mathrm{m}}, E_{\mathrm{z}}$.

In Nature, buckled spheroidal shells are usually found as a result of a prolonged period of fluid loss, either by drying or controlled expulsion, rather than an immediate collapse at a constant external pressure $[4,6,8,9]$. The controlled collapse of a spherical shell can be simulated numerically using a finite element solver coupling shell to hydrostatic fluid elements [24,25] (as opposed to mass-spring models published in earlier works $[4,13])$. The interaction between the shell and the encapsulated fluid corresponds to a fluid cavity constraint, given by $V-\bar{V}=0$, where $\bar{V}$ is the volume enclosed by the shell and $V$ is the volume of the fluid. The fluid volume is controlled by making its fluid density $\rho_{t}$ a function of simulation time $t$, using the relation $\rho_{t}=(1+\alpha t) \rho_{0}$, where $\alpha$ is the expansion coefficient and $\rho_{0}$ is the fluid density in the original unstressed configuration. Unlike previous studies on isotropic shells $[4,11]$, we limit ourselves to studying equilibrium postbuckling conformations by setting $\alpha=-0.1 \mathrm{~s}^{-1}$ and simulating $t=0 \rightarrow 10 \mathrm{~s}$. This low fluid volume compression rate ensures that our simulations traverse the energy landscape with a negligible probability of getting trapped in local minima.

The most intuitive way to prescribe orthotropic properties on the surface of a spherical shell is with reference to its poles. The elastic modulus $E_{\mathrm{m}}$ applies in the meridional direction, while $E_{\mathrm{z}}$ is defined in the zonal direction parallel to the equator as seen in Fig. 1(b). The meridians intersect at the poles, while the zonal lines remain parallel. This geometric setup implies that different buckling regimes occur when the ratio of the elastic moduli $\lambda=E_{\mathrm{z}} / E_{\mathrm{m}}$ is greater or less than unity. Details on the material properties and numerical implementation can be found in the Supplemental Material [26].

A variety of different postbuckled conformations are simulated in the parameter space of $R / h$ and $\lambda$ (Fig. 2). Three distinct phases of collapsed shells are identifiedsingle, symmetric biconcave double, and multiple indentations. The final post-buckled shapes are always found to be in the single indentation phase in the isotropic case $(\lambda=1)$, in agreement with some previous studies on spherical shells with zero spontaneous curvature $[10,13]$. In the isotropic case and for slender orthotropic shells

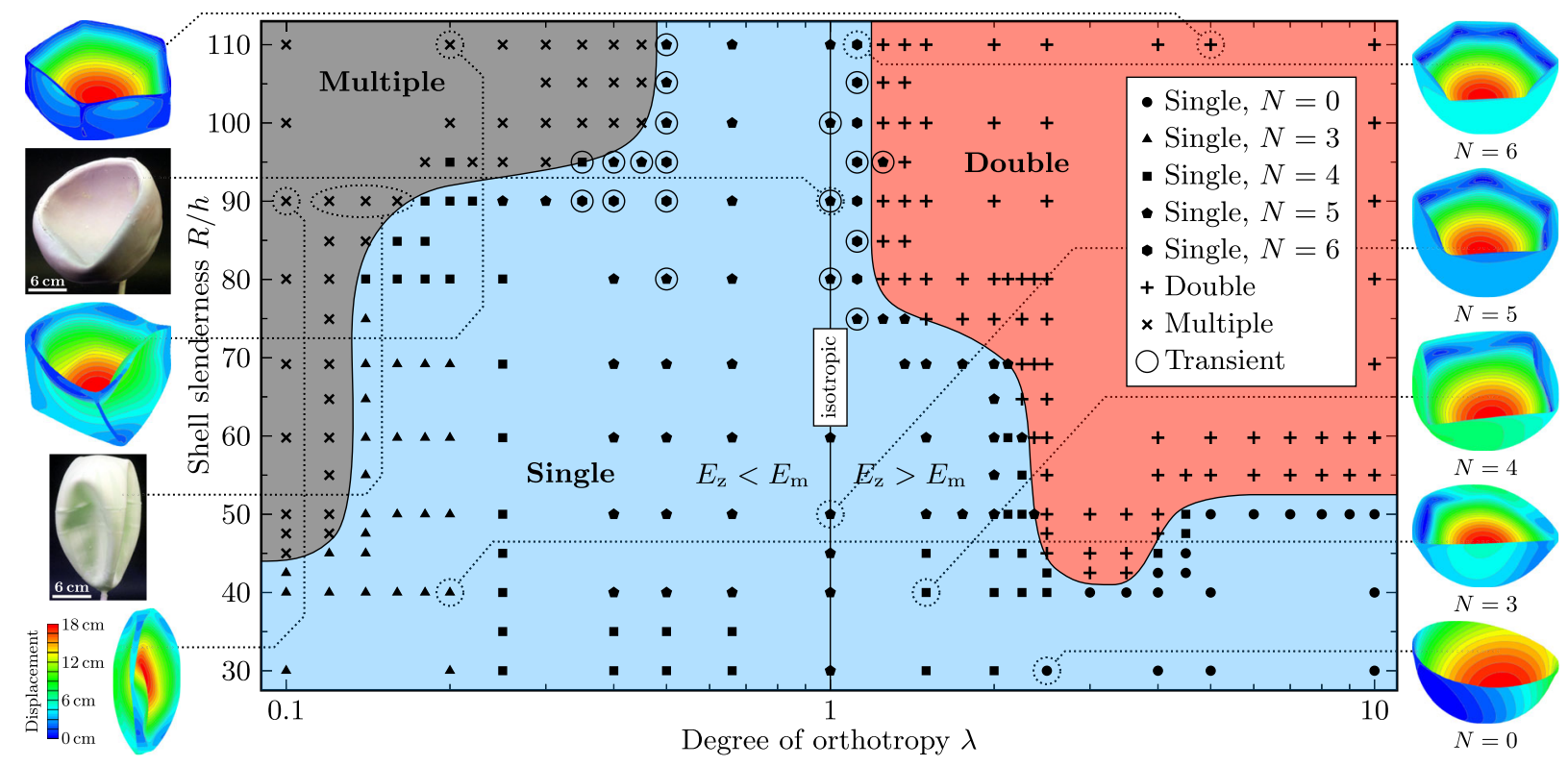

FIG. 2. Postbuckling conformations of spheres with varying degree of orthotropy $\lambda$ and slenderness $R / h\left(\Delta V / V_{0}=0.9\right)$. Three distinct buckling phases are distinguishable — single, biconcave double, and multiple indentations. The shape of the marker in the single indentation phase represents the number of corners, $N$, of its polygonal ridge. Solid lines surrounding a marker indicate transient behavior, implying that multiple indentations formed before relaxing to a single indentation. The colors on the simulation figures correspond to radial displacement. Movies of each of the three phases can be found in the Supplemental Material [26]. Experiments: side view of collapsed shells $(R / h=90)$ floating underwater for orthotropic $(\lambda=0.15)$ and isotropic $(\lambda=1)$ systems. 
$(R / h \approx 70-110)$ close to the boundaries of the single indentation phase, the structures can sometimes retain up to three indentations even with a low reduced volume fraction, $\Delta V / V_{0} \leq 0.9$ (where $V_{0}$ is the initial and $\Delta V$ is the lost fluid volume), before eventually undergoing a merging process to form a single indentation, thereby showcasing transient behavior in the system [13]. This low number of stable indentations (which we later confirm with our experiments) runs contrary to previous simulation studies for isotropic shells with much lower slenderness values $(R / h \leq 20)[4,11]$, which were based on a reduced Helfrich energy functional that does not account for Gaussian curvature rigidity.

As $\lambda$ deviates from unity, the post-buckling shapes become highly dependent on the slenderness. For thicker shells $(R / h \approx 30)$, the postbuckling conformations are still in the single indentation phase, but as the shells become thinner, two novel and distinct phases emerge. For $\lambda \approx$ $1.25-10$ and $70 \leq R / h \leq 110$, two indentations are initiated at opposite poles and grow inward at roughly the same rate until self-contact is realized. This is explained by the fact that the poles are converging points for meridional lines of lower modulus $\left(E_{\mathrm{m}}\right)$, which gives them a lower bending stiffness, making them the areas on the shell with the least resistance against indentations. This biconcave shape is characteristic of healthy red blood cells [32].

For slender shells with $\lambda \approx 0.1-0.5$, postbuckling conformations exhibit multiple indentations. In this phase, the shells initially morph into prolate spheroids with progressively growing ellipticity under fluid loss. This observation can be explained by the zonal lines having a lower $E_{\mathrm{z}}$ than the poles, resulting in the compression of the spherical shell along the equator and other zonal lines. Multiple spherical cap inversions then form, grow inwards, and remain robust even at very high $\Delta V / V_{0}$. The poles are the most stable areas on the spherical shell due to the convergence of the stiffer meridional lines, and the mean curvature $\kappa_{\mathrm{m}}$ is highest at the poles and lowest in the equatorial region. As the geometry-induced stiffness has been shown to be proportional to $\kappa_{\mathrm{m}}$ in spheroidal shells [33], indentations are expected to initiate around the flexible equatorial region. For highly orthotropic shells, with $\lambda \approx 0.1$ and $R / h=50-90$, the simulations follow this prediction, as a large initial change in the ellipticity drives the shell towards an elongated structure with three to four indentations along the meridional axis. This showcases a buckling pattern found in dehydrated tricolpate Brassica rapa pollen grains, whose folding has been shown to be guided by the composite intine of the cell wall $[34,35]$. However, for highly slender shells, with $R / h \approx 110$ and $\lambda \approx 0.25$, the change in ellipticity is much less significant, resulting in a very slight change in $\kappa_{\mathrm{m}}$ along the meridians. No correlation between patterns of equivalent stress and locations of the onset of indentations could be observed prior to buckling. This results in a cuboidlike structure with five to six indentations, similar to anisotropic gel phase vesicles in Ref. [11]. Given a buckled elastic homogeneous shell with a known thickness and diameter, one can infer the type of orthotropy $(\lambda<1$ or $\lambda>1)$ from the placement and number of indentations.

Secondary buckling modes also provide clues to $\lambda$, and are seen across the parameter space, generally along the periphery and perpendicular to the ridge of the initial indentation. These secondary effects give the ridge an equilateral polygonal shape with a number of corners, $N$, that increases with the slenderness due to the associated reduction in bending stiffness. Shells with $\lambda \ll 1$ usually have triangular-shaped ridges as the greater ellipticity of the spheroid makes it less energy efficient to form a square or other higher order polygons, unless the shell is very slender. A similar triangular-shaped ridge is found to be present in stomatocytes, which are formed due to defects in the membrane bilayer of red blood cells, which has been linked to membrane orthotropy $[8,36]$. For $\lambda \gg 1$, low $R / h$ values lead to the absence of any secondary buckling modes, resulting in a perfectly smooth spherical cap inversion, similar to thick isotropic shells in Ref. [10]. For $0.25<\lambda<4, \quad N$ generally increases with $R / h$ from $4 \rightarrow 5 \rightarrow 6$, with the exception of $\lambda=1$, which remains pentagonal in the simulated range $30<R / h<110$.

The evolution of the strain energy $U$ gives further insight into shell collapse dynamics. At very high reduced volume fractions $\left(\Delta V / V_{0}>0.9\right), U$ is shown to increase significantly due to self-contact between the indentation(s) [Fig. 3(a)]. The parameters $\lambda=0.5$ and $R / h=110$ represent the transient case, where for a majority of simulation runs, a large drop in $U$ is seen due to a reduction in the number of indentations at high $\Delta V / V_{0}$. For moderate volume fractions $\left(0.1<\Delta V / V_{0}<0.6\right)$, depending on $\lambda$, we find that $U \sim\left(\Delta V / V_{0}\right)^{\beta}$ with an exponent of $\beta=$ $0.72 \pm 0.02$ [Fig. 3(b)], which is very close to previous work on isotropic shells [13]. The strain energy in the region $\Delta V / V_{0}=0.1 \rightarrow 0.6$ is always largest at $\lambda=1$ and reduces as $\lambda$ deviates from $1 . \lambda<0.5$ has higher $U$ than $\lambda>2.0$. This is expected, as $U$ has been shown to scale with the number of indentations with an exponent of 0.25 [17]. Regardless of the shell properties, at $\Delta V / V_{0}<0.1$, there is a peak in $U$ corresponding to the initial spherical cap inversion. This increase in $U$ scales quadratically with $\Delta V / V_{0}$ [Fig. 3(c)]. At $\lambda=1$, the peak occurs at a relatively high $\Delta V / V_{0}$ compared to moderately orthotropic materials $(0.5<\lambda<2.0)$, due to the inherent stability of the homogeneous isotropic shell. As $\lambda$ deviates further from unity, the peak shifts back to larger $\Delta V / V_{0}$ values, and in the case of $\lambda=0.1-0.4$, even exceeds that of the isotropic case. While this might seem counterintuitive, it can be explained by the effect of the geometric transformation of the shell towards ellipticity outweighing the loss in bending stiffness caused by the high degree of orthotropy. This effect is more pronounced for $\lambda<0.5$, as 

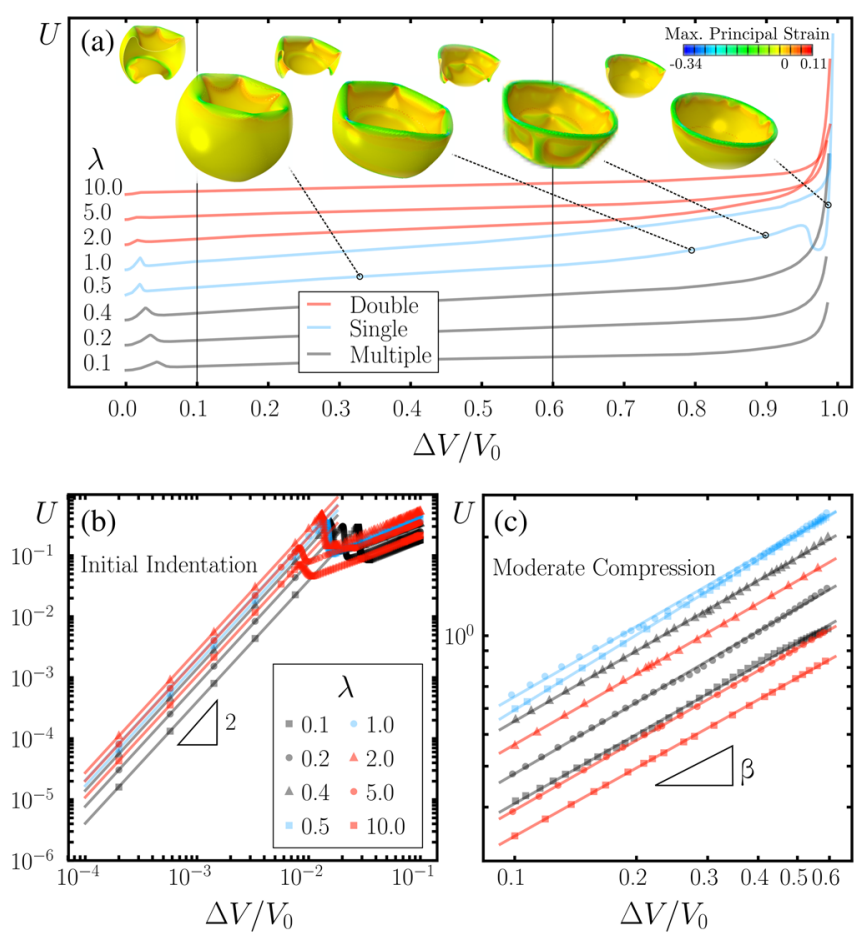

FIG. 3. (a) Evolution of the strain energy $U$ (Joule) for different $\lambda$ at fixed slenderness $R / h=110$. The colors represent the single, biconcave double, and multiple indentation phases from Fig. 2. Note that curves are vertically shifted for better visibility. Snapshots and their cuts are shown for the transient buckling case $(\lambda=0.5)$ at specific reduced volume fractions. (b) $U$ scales quadratically with $\Delta V / V_{0}$ before the onset of the first indentation. (c) $U$ scales with $\Delta V / V_{0}$ as a power law with exponent $\beta=0.72 \pm 0.02$ in the region $0.1 \leq \Delta V / V_{0} \leq 0.6$.

these structures undergo larger geometric changes than for $\lambda>2.0$, allowing the $\lambda<0.5$ shells to withstand higher compressive stresses without collapsing.

To support our numerical findings with experimental evidence, we reproduced the volume-controlled collapse with a versatile technique for fabricating shells [37,38]. The procedure consists of pouring a silicone-based liquid polymer solution onto a steel sphere, resulting in hemispherical shells of relatively predictable thickness after the uncured polymer has drained. To create a spherical shell, two hemispherical caps ( $R$ of $110 \mathrm{~mm}$ and $h$ of $1.2 \pm 0.06 \mathrm{~mm}$ ) are produced separately and glued together at the equator (Fig. S1). In the orthotropic case, strips of thin PTFE film are glued on in the meridional direction to provide fiber reinforcement. The effect of the stiffer PTFE results in an orthotropic shell with a $\lambda$ of 0.15 using equivalent stiffness models for composites [39]. These spherical shells are then filled with water through a valve and placed inside a water bath. In the case of the isotropic shell, ejecting the encapsulated water at different rates (over 1-100 s) always showcases a robust single indentation, with $N=4-5$ (Fig. 2). The orthotropic case yields a shape very similar to the elongated structure (similar to tricolpate

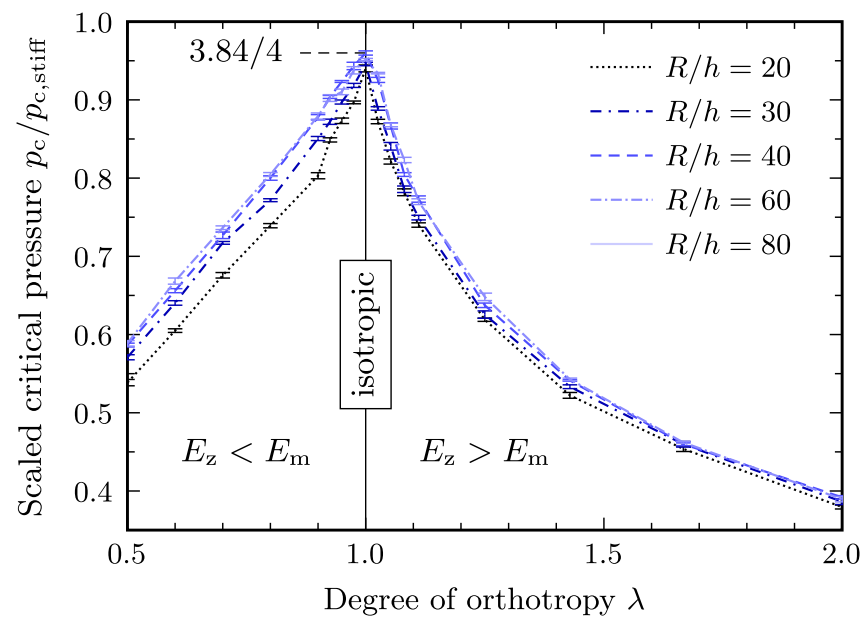

FIG. 4. Scaling of the critical buckling pressure with $\lambda$ and $R / h$. Each data point represents the average over five independent simulations; error bars denote statistical errors.

Brassica rapa pollen) found in the simulated multiple indentations phase.

Studying the change in critical pressure $p_{\mathrm{c}}$ for shells with varying $R / h$ and $\lambda$ provides a quantitative description of their structural stability [13]. Since orthotropic extensions of Eq. (1) do not exist for spherical or ellipsoidal shells to the best of our knowledge [40], our analysis of $p_{\mathrm{c}}$ for anisotropic shells can only be validated compared to the theoretical $p_{\mathrm{ci}}$. To simulate rapid collapse, subdivision surface shell elements within a dynamic time integration framework [25,41-43] are used to ensure a high degree of accuracy.

The numerical results confirm that $p_{\mathrm{c}}$ of the orthotropic spherical shells remains approximately proportional to $(R / h)^{-2}$ as in Eq. (1) as shown in Fig. 4. All pressure data approximately collapse onto a master curve after scaling by

$$
p_{\mathrm{c}, \mathrm{stiff}}=\frac{4 \max \left\{E_{\mathrm{m}}, E_{\mathrm{z}}\right\}}{\sqrt{12\left(1-\min \left\{\nu_{\mathrm{zm}}, \nu_{\mathrm{mz}}\right\}^{2}\right)}}\left(\frac{h}{R}\right)^{2}
$$

The collapse converges in the membrane limit $R / h \rightarrow \infty$. $p_{\mathrm{c}}$ is scaled by $p_{\mathrm{c}, \text { stiff }}$ because in the orthotropic case, buckling can only be initiated when the bending energy barrier in both principal tangential directions is exceeded, making the critical pressure governed by the stiffer of the two in first order. This is consistent with the behavior of other types of material tuning [15].

The dependence of $p_{\mathrm{c}}$ on $\lambda$ reveals a distinct difference between the two orthotropic regimes $\lambda<1$ and $\lambda>1$. The theoretical prefactor of 4 (3.84 \pm 0.03 in the simulations) decreases as $\lambda$ deviates from 1 . The regime with $\lambda<1$ always provides larger prefactors than $\lambda>1$ for the same logarithmic distance $|\log \lambda|$, as is expected from the controlled collapse analysis. While for $\lambda<1$ the critical 
pressure drops nearly linearly with the degree of orthotropy for sufficiently thin shells in the examined range, the scaling is nonlinear for $\lambda>1$ and does not appear to follow a simple functional relationship. A part of the observed nonlinearity in $\lambda$ stems from the nonlinear deformation of orthotropic spheres into spheroids under compression [40].

Our Letter shows that material orthotropy and shell slenderness can be tuned to achieve three distinct regimes of postbuckled shapes. This suggests that the shapes of red blood cells and Brassica rapa pollen occurring in Nature can now be examined through the lens of anisotropic properties, yielding insight into their structure-function relationship $[6,8,9]$. We also find that strongly orthotropic materials can be more stable under controlled collapse than isotropic structures, despite having lower equivalent stiffness, resulting in potential applications in the fabrication of functional colloids. Finally, the variability of the scaling behavior of the critical pressure with the type of orthotropy $(\lambda<1$ or $\lambda>1)$ poses an interesting query that requires further theoretical insight.

The authors would like to thank Benoit Roman for his assistance in shell fabrication and Alessandro Leonardi for helpful suggestions. Furthermore, the authors acknowledge support from the Research and Technology Development Project MecanX: Physics-Based Models of Growing Plant Cells using Multi-Scale Sensor Feedback granted under SystemsX.ch by the Swiss National Science Foundation, the Advanced Grant No. 319968-FlowCCS granted by the European Research Council (ERC) and from ETH Zürich by ETHIIRA Grant No. ETH-03 10-3. H. J. H. would like to thank CAPES and FUNCAP.

*Corresponding author. gmunglani@gmail.com

[1] R. T. Cole, An analysis of the general instability of eccentrically stiffened complete spherical shells under uniform pressure, Ph.D. thesis, Georgia Institute of Technology, 1969.

[2] D. Bushnell, Buckling of shells-pitfall for designers, AIAA J. 19, 1183 (1981).

[3] C. Gao, E. Donath, S. Moya, V. Dudnik, and H. Möhwald, Elasticity of hollow polyelectrolyte capsules prepared by the layer-by-layer technique, Eur. Phys. J. E 5, 21 (2001).

[4] C. Quilliet, C. Zoldesi, C. Riera, A. Van Blaaderen, and A. Imhof, Anisotropic colloids through non-trivial buckling, Eur. Phys. J. E 27, 13 (2008).

[5] N. Tsapis, E. R. Dufresne, S. S. Sinha, C. S. Riera, J. W. Hutchinson, L. Mahadevan, and D. A. Weitz, Onset of Buckling in Drying Droplets of Colloidal Suspensions, Phys. Rev. Lett. 94, 018302 (2005).

[6] E. Katifori, S. Alben, E. Cerda, D. R. Nelson, and J. Dumais, Foldable structures and the natural design of pollen grains, Proc. Natl. Acad. Sci. U.S.A. 107, 7635 (2010).
[7] W. W. Payne, Observations of harmomegathy in pollen of Anthophyta, Grana 12, 93 (1972).

[8] G. H. W. Lim, M. Wortis, and R. Mukhopadhyay, Stomatocyte-discocyte-echinocyte sequence of the human red blood cell: Evidence for the bilayer-couple hypothesis from membrane mechanics, Proc. Natl. Acad. Sci. U.S.A. 99, 16766 (2002).

[9] Y. Park, C. A. Best, K. Badizadegan, R. R. Dasari, M. S. Feld, T. Kuriabova, M. L. Henle, A. J. Levine, and G. Popescu, Measurement of red blood cell mechanics during morphological changes, Proc. Natl. Acad. Sci. U.S.A. 107, 6731 (2010).

[10] C. Quilliet, Numerical deflation of beach balls with various Poisson's ratios: From sphere to bowl's shape, Eur. Phys. J. E 35, 48 (2012).

[11] F. Quemeneur, C. Quilliet, M. Faivre, A. Viallat, and B. Pépin-Donat, Gel Phase Vesicles Buckle into Specific Shapes, Phys. Rev. Lett. 108, 108303 (2012).

[12] C.Q. Ru, Buckling of empty spherical viruses under external pressure, J. Appl. Phys. 105, 124701 (2009).

[13] G. A. Vliegenthart and G. Gompper, Compression, crumpling and collapse of spherical shells and capsules, New J. Phys. 13, 045020 (2011).

[14] S. S. Datta, S.-H. Kim, J. Paulose, A. Abbaspourrad, D. R. Nelson, and D. A. Weitz, Delayed Buckling and Guided Folding of Inhomogeneous Capsules, Phys. Rev. Lett. 109, 134302 (2012).

[15] J. Paulose and D. R. Nelson, Buckling pathways in spherical shells with soft spots, Soft Matter 9, 8227 (2013).

[16] L. Pauchard, Invagination during the collapse of an inhomogeneous spheroidal shell, Europhys. Lett. 66, 667 (2004).

[17] C. Quilliet, Depressions at the surface of an elastic spherical shell submitted to external pressure, Phys. Rev. E 74, 046608 (2006).

[18] S. Knoche and J. Kierfeld, Buckling of spherical capsules, Phys. Rev. E 84, 046608 (2011).

[19] S. Knoche and J. Kierfeld, Osmotic buckling of spherical capsules, Soft Matter 10, 8358 (2014).

[20] T. Baskin, Anisotropic expansion of the plant cell wall, Annu. Rev. Cell Dev. Biol. 21, 203 (2005).

[21] S. Kerstens, W. F. Decraemer, and J.-P. Verbelen, Cell walls at the plant surface behave mechanically like fiberreinforced composite materials, Plant Physiol. 127, 381 (2001).

[22] R. Zoelly, Über ein Knickungsproblem an der Kugelschale, Ph.D. thesis, ETH Zürich, 1915.

[23] T. von Kármán and H.-S. Tsien, The buckling of spherical shells by external pressure, J. Aeronaut. Sci. 7, 43 (1939).

[24] ABAQUS/CAE User's Guide, Version 6.14, 2014.

[25] R. Vetter, N. Stoop, T. Jenni, F. K. Wittel, and H. J. Herrmann, Subdivision shell elements with anisotropic growth, Int. J. Numer. Methods Eng. 95, 791 (2013).

[26] See Supplemental Material at http://link.aps.org/ supplemental/10.1103/PhysRevLett.123.058002 for the material properties, numerical implementation, and shell fabrication, which includes Refs. [27-31].

[27] B. Budiansky and J. Sanders, The "best" first-order linear shell theory, Progress Applied Mechanics, The Prager Anniversary Volume (Macmillan, London, 1963), pp. 129-140. 
[28] Y. Li and J. Barbič, in Proceedings of the ACM SIGGRAPH/ Eurographics Symposium on Computer Animation, SCA '14 (Eurographics Association, Aire-la-Ville, Switzerland, 2014), pp. 41-46.

[29] M. T. Huber, The theory of crosswise reinforced ferroconcrete slabs and its applications to various important constructional problems involving rectangular slabs, Bauingenieur 4, 354 (1923).

[30] H. A. Mang, Symmetricability of pressure stiffness matrices for shells with loaded free edges, Int. J. Numer. Methods Eng. 15, 981 (1980).

[31] J. T. Oden and E. B. Pires, Nonlocal and nonlinear friction laws and variational principles for contact problems in elasticity, J. Appl. Mech. 50, 67 (1983).

[32] Y. Park, C. A. Best, K. Badizadegan, R. R. Dasari, M. S. Feld, T. Kuriabova, M. L. Henle, A. J. Levine, and G. Popescu, Metabolic remodeling of the human red blood cell membrane, Proc. Natl. Acad. Sci. U.S.A. 107, 1289 (2010).

[33] A. Lazarus, H. C. B. Florijn, and P. M. Reis, GeometryInduced Rigidity in Nonspherical Pressurized Elastic Shells, Phys. Rev. Lett. 109, 144301 (2012).

[34] C. Ferguson, T. T. Teeri, M. Siika-aho, S. M. Read, and A. Bacic, Location of cellulose and callose in pollen tubes and grains of Nicotiana tabacum, Planta 206, 452 (1998).

[35] A. B. Kirkpatrick and H. A. Owen, Observation of early pollen exine patterning by scanning electron microscopy, Microsc. Microanal. 19, 134 (2013).
[36] T. Fischer, C. Haest, M. Stöhr-Liesen, H. SchmidSchönbein, and R. Skalak, The stress-free shape of the red blood cell membrane, Biophys. J. 34, 409 (1981).

[37] A. Lee, P.-T. Brun, J. Marthelot, G. Balestra, F. Gallaire, and P. M. Reis, Fabrication of slender elastic shells by the coating of curved surfaces, Nat. Commun. 7, 11155 (2016).

[38] E. Siéfert, E. Reyssat, J. Bico, and B. Roman, Bio-inspired pneumatic shape-morphing elastomers, Nat. Mater. 18, 24 (2019).

[39] F. M. I. Xia, Yuying, and E. I. Saavedra Flores, Equivalent models of corrugated panels, Int. J. Solids Struct. 49, 1453 (2012).

[40] S. N. Krivoshapko, Research on general and axisymmetric ellipsoidal shells used as domes, pressure vessels, and tanks, Appl. Mech. Rev. 60, 336 (2007).

[41] F. Cirak, M. Ortiz, and P. Schröder, Subdivision surfaces: a new paradigm for thin-shell finite-element analysis, Int. J. Numer. Methods Eng. 47, 2039 (2000).

[42] R. Vetter, N. Stoop, F. K. Wittel, and H. J. Herrmann, Simulating thin sheets: Buckling, wrinkling, folding and growth, J. Phys. Conf. Ser. 487, 012012 (2014).

[43] G. Munglani, R. Vetter, F. K. Wittel, and H. J. Herrmann, Orthotropic rotation-free thin shell elements, Comput. Mech. 56, 785 (2015). 